\title{
An In Vitro Culture System That Supports Robust Expansion and Maintenance of In Vivo Engraftment Capabilities for Myogenic Progenitor Cells from Adult Mice
}

\author{
Zhan Wang,, Daniel Cheung, ${ }^{2}$ Yu Zhou, Changjie Han,, Colin Fennelly, Tracy Criswell,, ${ }^{1,3}$ and Shay Soker ${ }^{1,3}$
}

\begin{abstract}
Muscle cell therapy and tissue engineering require large numbers of functional muscle precursor/progenitor cells (MPCs), making the in vitro expansion of MPCs a critical step for these applications. The cells must maintain their myogenic properties upon robust expansion, especially for cellular therapy applications, in order to achieve efficacious treatment. A major obstacle associated with MPCs expansion is the loss of "stemness," or regenerative capacity, of freshly isolated cells, presumably due to the absence of the native cellular niches. In the current study, we developed an in vitro system that allowed for long-term culture and massive expansion of murine MPCs (mMPCs) with the preservation of myogenic regeneration capabilities. Long term in vitro expanded mMPC expressed the myogenic stem cell markers Pax3 and Pax7 and formed spontaneously contracting myotubes. Furthermore, expanded mMPC injected into the tibialis anterior muscle of nude mice engrafted and formed myofibers. Collectively, the method developed in this study can be potentially adapted for the expansion of human MPCs to high enough numbers for treatment of muscle injuries in human patients.
\end{abstract}

Key words: cell culture; cell transplantation; cellular therapy; muscle stem cell

\section{Introduction}

O BTAINING SUFFICIENT NUMBER of functional muscle stem/progenitor cells (MPCs) is essential for the success of many applications, including tissue engineering, drug screening, ${ }^{1,2}$ and cell therapy. ${ }^{3-6}$ Cell therapy for example, requires $10^{6}$ to $10^{9}$ MPCs for transplantation to repair diseased muscle or large volumetric muscle loss. ${ }^{6-8}$ In vitro cell expansion, or cell culture, is the most practical way to achieve such large numbers of cells. On the other hand, it is critical that MPCs retain their myogenic properties during culture, including contractility and the ability to engraft, in order to ensure success of the above-mentioned applications. ${ }^{1-6,9}$

In vivo, the fate of the MPCs is closely regulated by the local tissue microenvironment, or the stem cell niche. The niche regulates muscle stem cell fate through multiple extrinsic cues, including soluble secreted factors, local extracellular matrix (ECM) proteins and muscle mechanotransduction (reviewed previously ${ }^{10-13}$ ). Therefore, the rapid loss of myogenic "stemness" in cultured MPCs may be due to the absence of the native tissue niche. ${ }^{4,14,15}$ For this reason, to date, the best outcome for muscle stem cell therapy in preclinical animals models has been from either the use of single muscle fiber implantation, ${ }^{3}$ which provides satellite cells still embodied within their physical niche, or from injection of freshly isolated MPCs,,${ }^{4,16,17}$ which minimizes the in vitro manipulation to the cells. Despite some early success of these methods to produce myogenic cells that can aid in the regenerative process in preclinical models, they are not appropriate for clinical use, due to the low number of cells available for therapy. ${ }^{3,8,16}$ On the other hand, recent studies using embryonic stem cells ${ }^{18-20}$ and adult muscle stem cells ${ }^{14}$ have suggested the possibility of maintaining "stemness" through the provision of niche-associated components. These studies suggest that the provision of extrinsic cues, especially growth factors and ECM proteins, may provide support to maintain stem cell myogenic and self-renewal properties.

In the present study, we tested several culture conditions to identify appropriate conditions for long-term MPC expansion in culture. A simple combination of myogenic cell media and Matrigel ${ }^{\mathrm{TM}}$-coated substratum supported expansion of murine MPCs (mMPCs) for up to 25 passages, as well as expression of the muscle stem cell markers Pax3 and Pax7 and maintenance of myogenic properties including the ability to form myotubes and myofibers, in vitro and in vivo, respectively.

\footnotetext{
${ }^{1}$ Wake Forest Institute for Regenerative Medicine, ${ }^{3}$ Wake Forest School of Medicine, Winston-Salem, North Carolina.

${ }^{2}$ Oregon State University, School of Chemical, Biological, and Environmental Engineering, Corvallis, Oregon.
} 


\section{Materials and Methods}

\section{mMPC isolation, culture and expansion}

Tissues were collected from 8- to 12-week-old green fluorescent protein (GFP) transgenic mice (C57BL/Ka-b-actinEGFP), including the tibialis anterior (TA), soleus, gastrocnemius, quadriceps, and triceps muscles. Fat and connective tissues were carefully dissected from the muscle prior to digestion. Muscle tissues were digested in $0.2 \%$ collagenase type I (Worthington Biochemical Corporation, Lakewood, NJ) for 1.5 hours, then neutralized with fetal bovine serum (FBS). Digested muscle tissues were spun down and then resuspended in myogenic medium (Myo medium), consisting of Dulbecco's modified Eagle's medium (DMEM) $+20 \%$ FBS $+10 \%$ horse serum $+1 \%$ chicken embryo extract (CEE) (Sera Laboratory, United Kingdom) $+1 \%$ antibiotic/ antimycotic solution (HyClone, US).

Prior to cell plating, tissue culture plates were coated with 1:200 phosphate-buffered saline (PBS)-diluted $\left(50 \mu \mathrm{L} / \mathrm{cm}^{2}\right)$ Matrigel $^{\mathrm{TM}}$ (BD 354234, BD Biosciences, US) at $37^{\circ} \mathrm{C}$ overnight. Immediately before cell seeding, the Matrigel solution was aspirated without further washing. Digested tissue $(1 \mathrm{~g} /$ $50 \mathrm{~cm}^{2}$ ) was directly seeded on the coated culture dishes in Myo medium ( $10 \mathrm{~mL} / \mathrm{g}$ tissue), and cultured in a humidified $5 \% \mathrm{CO}_{2}$ atmosphere at $37^{\circ} \mathrm{C}$. Seventy-two hours after cell plating, all residual tissue and culture medium was removed and replenished with fresh Myo medium. Cells were cultured in Myo medium until passaging. In each passage, after 3-5 days in culture cells were trypsinized and harvested at $60 \%$ confluence then reseeded at 5000 cells $/ \mathrm{cm}^{2}$ on 1:200 Matrigel-coated tissue culture plates with Myo medium $(0.2 \mathrm{~mL} /$ $\mathrm{cm}^{2}$ ) (Supplementary Fig. S1). Myo medium and 1:200 Matrigel coating were used for all following cell passaging and differentiation assay (Supplementary Fig. S1). Cultured cells may have fibroblast-like cells contamination in early passages. Continuing splitting following the described protocol will lead to a MPC-dominant culture and eliminate fibroblast-like cell contamination. Typically it took about 35 and 80 days to reach the passage 10 and 25 , respectively.

\section{Human MPC culture}

Usage of discarded human tissue was approved by the Wake Forest School of Medicine IRB. Human MPC (hMPC) in vitro culture was performed as described previously, ${ }^{21}$ with minor modifications. Briefly, discarded human skeletal muscle tissue from hip replacement surgeries was rinsed with sterilized PBS and digested with collagenase Type I $0.2 \%$ (w/v) (Worthington Biochemical) and dispase $0.4 \%(\mathrm{w} / \mathrm{v})$ (Gibco). Digested tissue was seeded on collagen type Icoated tissue culture plates, in DMEM/F12 nutrient mix (1:1) supplemented with $18 \% \mathrm{FBS}, 5 \mu \mathrm{g} / \mathrm{mL}$ gentamicin, $10 \mathrm{ng} / \mathrm{mL}$ human epidermal growth factor, $1 \mathrm{ng} / \mathrm{mL}$ human basic fibroblast growth factor, $10 \mu \mathrm{g} / \mathrm{mL}$ human insulin, and $0.4 \mu \mathrm{g} / \mathrm{mL}$ dexamethasone. After two passages, hMPCs were cultured in the same medium on noncoated tissue culture plates.

\section{Mouse strains}

Mouse strains were bred and maintained at Wake Forest University in compliance with the Wake Forest University
Institutional Animal Care and Use Committee and National Institutes of Health (NIH) guidelines. Male and female (812 weeks of age) GFP-transgenic mice (C57BL/Ka-bactin-EGFP) were purchased from Jackson Laboratories ${ }^{22}$ and used as the source of mMPCs. Female (8-12 weeks of age) nude mice $(\mathrm{Nu} / \mathrm{Nu})$, purchased from Harlan Laboratories, were used for cell transplantation studies.

\section{Cardiotoxin tissue injury}

$\mathrm{Nu} / \mathrm{Nu}$ mice (8-12 weeks of age) were anesthetized and injected intramuscularly with $30 \mu \mathrm{L}(0.03 \mathrm{mg} / \mathrm{mL})$ Naja mossambica cardiotoxin (Sigma) into the TA muscle 1 day prior to cell transplantation, as reported before. ${ }^{16}$ Mouse $\mathrm{GFP}^{+}$ MPCs $\left(1 \times 10^{5}\right)$ in $20 \mu \mathrm{L}$ of 1:5 PBS-diluted Matrigel were injected into the injured TA muscle via a Hamilton syringe. To ensure accurate and consistent cell injections, an incision was made through the skin and fascia of recipient mice at the lateral aspect of the lower leg, and the wound was sutured closed after injection. TA muscles were harvested and analyzed 4 weeks after cell injection.

\section{Myotube formation assays}

Murine MPCs were plated at a density of 5000 cells $/ \mathrm{cm}^{2}$ on a 1:200 dilution of Matrigel-coated plates in Myo medium $\left(0.2 \mathrm{~mL} / \mathrm{cm}^{2}\right)$. Cells were allowed to grow to high density, which resulted in spontaneous fusion into multinucleated myotubes. No medium change was required before imaging. Images of the cultures were obtained 7 days after plating. ImageJ software (NIH, Bethesda, MD) was used to quantify total myotube length and percentage of myotubes with more than five nuclei.

\section{Tissue analyses}

Injured TA muscles were harvested 28 days after injury and processed for histological analyses. Tissue samples were immediately embedded into Optimal Cutting Temperature compound (Tissue-Tek) and frozen in liquid nitrogen. Serial frozen tissue slices (8- $\mu$ m thickness) were prepared. GFP was detected microscopically by epifluorescence and immunohistochemistry staining. The autofluorescence of the muscle tissue was separated from the GFP signal at $509 \mathrm{~nm}^{16,23,24}$ by using Nuance EX multispectral tissue imaging system (PerkinElmer) and Olympus VS110 equipped with SEMROCK filter (Olympus). One hundred to 200 serial sections were used to determine the number of donor $\mathrm{GFP}^{+}$ myofibers. Data were presented as the mean $( \pm \mathrm{SD})$ number of $\mathrm{GFP}^{+}$myofibers in the section that contained the most $\mathrm{GFP}^{+}$myofibers. The engraftment efficiency was quantified by calculating the regenerative index (RI) as the number of donor-derived myofibers per $10^{5}$ transplanted cells. ${ }^{25}$

\section{Immunostaining}

Tissue and cells were fixed in $4 \%$ paraformaldehyde, permeabilized with $0.1 \%$ Triton $\mathrm{X}-100$, blocked with protein blocker (Dako, X090930), and incubated with primary antibodies for $1.5 \mathrm{~h}$ at room temperature, followed by secondary antibodies for $30 \mathrm{~min}$ at room temperature.

Primary antibodies. Mouse anti-human nuclei (1:50) (Millipore MAB1281), Pax7 (1:500), Pax3 (1:500), Myogenin 
(1:500) (Developmental Studies Hybridoma Bank), Laminin (C-20) (1:200) (Sigma), Laminin (1:400) (Abcam), MyoD (C-20) (1:100), Desmin (RD301) (1:50), and GFP (FL) (1:50) (Santa Cruz).

Secondary antibodies and other reagents. Goat anti-rabbit $\operatorname{IgG}(1: 200)$ (FITC or TexRed conjugate [Vector]), goat antimouse IgG (FITC or TexRed conjugate [Vector]), biotinylated goat anti-rabbit IgG (1:100) (Vector), biotinylated goat anti-mouse $\operatorname{IgG}(1: 100)$ (Vector),VECTASTAIN Elite ABC Reagent, R.T.U. (Vector PK-7100), and ImmPACT DAB Peroxidase Substrate (Vector SK-4105).

\section{Statistical analysis}

Difference was compared with Student's $t$-test or one-way ANOVA using GraphPad Prism software version 3.0a (GraphPad Software, Inc., La Jolla, CA); $p<0.05$ was considered statistically significant.

\section{Results \\ Optimization of in vitro cell culture conditions for $M M P C$ expansion}

The overarching hypothesis for this study is that specific combination of growth factors and adhesive proteins can preserve the myogenic capacity of MPCs during long-term cell culture expansion. We tested five different culture media and ECM coating (Fig. 1A, B) for supporting mMPC in vitro expansion. The culture conditions being tested were the following: Condition I: Myo medium + nondiluted Matrigel coated dishes; Condition II: Myo medium + 1:200 diluted Matrigel coated dishes; Condition III: Myo medium + uncoated tissue culture dishes; Condition IV: DMEM + 10\% FBS + uncoated dishes; and Condition V: DMEM + 10\% FBS + 1:200 diluted Matrigel-coated dishes. As a first screen, we examined the ability of the cultured mMPCs to fuse and form myotubes in vitro (Fig. 1A, B). All culture conditions supported mMPC (passage 0) fusion into myotubes, but cells grown in Myo medium on Matrigel (Conditions I and II) demonstrated significantly higher total length of myotubes (Fig. 1A, B), suggesting that the combination of Myo medium and Matrigel coating were beneficial for mMPC differentiation.

Since myotubes are generated from single-nucleated mMPCs, the myotube formation results suggested that Myo medium and Matrigel coating also increased mMPC proliferation. To confirm this hypothesis, we measured the number of mMPCs expanded in vitro under these culture conditions. The expanded mMPCs showed exponential growth under Condition II, whereas mMPCs cultured under the other conditions failed to proliferate at passage 2 (Fig. 1C). For practical reasons we chose to use culture condition II, with 1:200 dilution of Matrigel, as our optimal condition in the remaining experiments, because it had a similar outcome as undiluted Matrigel. Long-term mMPC expansion under Condition II enabled us to exponentially expand mMPCs from an initial $3 \times 10^{6}$ cells at passage 0 , to $1 \times 10^{12}$ cells at passage 10 , while maintaining typical mMPC morphology of small spindle-shaped cells or elongated, single-nucleated cells (Fig. 1D, F), which implied stable phenotype during long-term cell culture. Even after long-term expansion, to passages 10 (P10) and 25 (P25), the MPCs showed a homogeneous, diploid DNA content in the G1 (prereplicative) phase of the cell cycle (Fig. 1E). This result implies that the G1 and G2 cell-cycle checkpoints appeared intact and suggests normal ploidy of the MPCs after long-term expansion.

\section{In vitro expanded mMPC exhibits muscle stem cell phenotype and differentiation capacity}

Pax 3 and Pax 7 are key transcription factors regulating skeletal muscle development ${ }^{26,27}$ and regeneration. ${ }^{28}$ To evaluate whether our culture method is capable of maintaining these myogenic markers during extensive in vitro expansion, we measured the expression of Pax7 and Pax 3 in passages 0 to 4 (P0-P4) and 20 to 25 (P20-25) of mMPCs. Typical images, as shown in Fig. 2A indicates the presence of $\mathrm{Pax} 7^{+}$and Pax $3^{+}$mMPCs at passages 0 and 25. Figure 2B shows the quantification of Pax $7^{+}$mMPCs at both low and high passages and determined that $40 \%-60 \%$ of cultured mMPCs were positive for Pax7 expression, indicating that our culture conditions preserved the myogenic properties of mMPCs. In contrast, under the other culture conditions (Conditions III, IV, and V) no more than $10 \%$ of cultured mMPCs expressed Pax7 (Supplementary Fig. S2A, B) at passage 0 . As a comparison, we also examined Pax7 and Pax3 expression in several cultures of hMPCs that were cultured in growth factor contained medium in collage I-coated tissue culture dishes, as indicated in methods part. Among nine samples tested, only one sample showed Pax3 expression and four showed Pax7 expression. In the hMPC culture in which we observed Pax3 and Pax7 expression, only 3.5\% and $4.3 \%$ of the cells were positive for Pax3 and Pax7, respectively, suggesting the loss of myogenic "stemness" under nonoptimal culture conditions (Supplementary Fig. S3A, B).

We further examined the expression of Myf5, MyoD, and Myogenin in order to determine the differentiation state of mMPCs expanded long term under our culture conditions. As shown in Figure 2C, D, about $90 \%$ and $70 \%$ of the mMPCs expressed the early myogenic marker Myf5 and the mid-differentiation myogenic marker MyoD, respectively. On the other hand, only about $40 \%$ of mMPCs expressed the late differentiation marker Myogenin. These data suggest that our optimized culture conditions are capable of maintaining the majority of expanded mMPCs at the undifferentiated stage of muscle progenitors.

Myotube formation, as determined by the presence of multinucleated myotubes, is a measure to determine mMPCs differentiation capacity and can also be used to determine myotube maturity. In vitro expanded mMPCs showed cell fusion and myotube formation for up to passage 25 (Fig. 3A). The myotubes exhibited a striated appearance, suggesting proper sarcomeric organization, and expressed Desmin, a sarcomeric intermediate filament protein (Fig 3B). We subsequently quantified the myotube forming capacities at different passages. About $60 \%-80 \%$ of multinucleated myotubes had five or more nuclei per myotube, consistently from passage 1 to 11, and in passage 25 (Fig 3C), implying the maintenance of in vitro myogenic potential during longterm cell expansion. Furthermore, under the optimized culture conditions (Condition II), we were able to observe spontaneous contraction of the myotubes (Supplementary Movies A-C). 

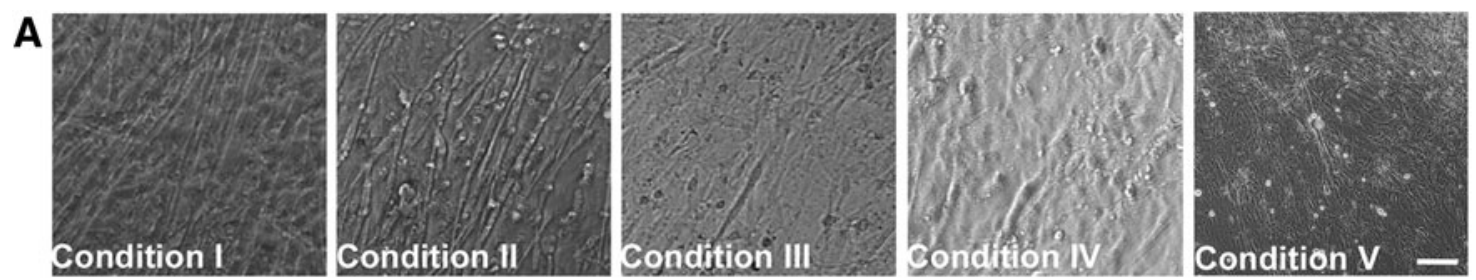

B

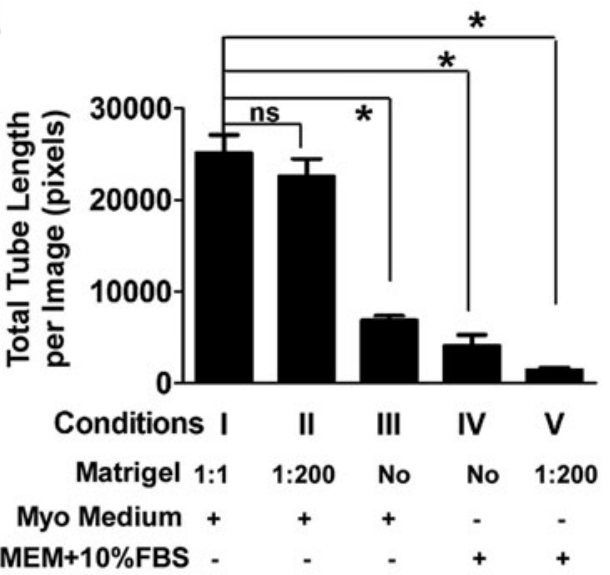

D

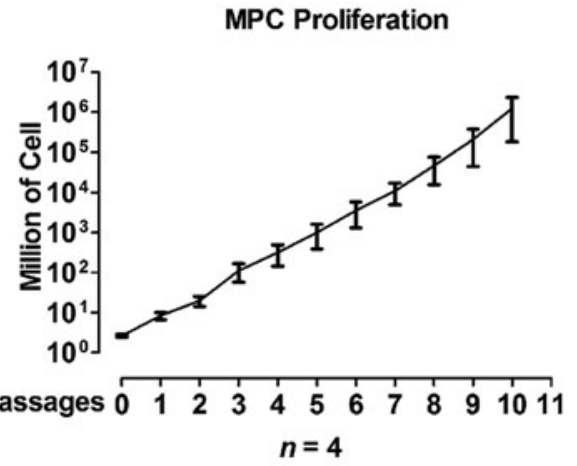

F

P0

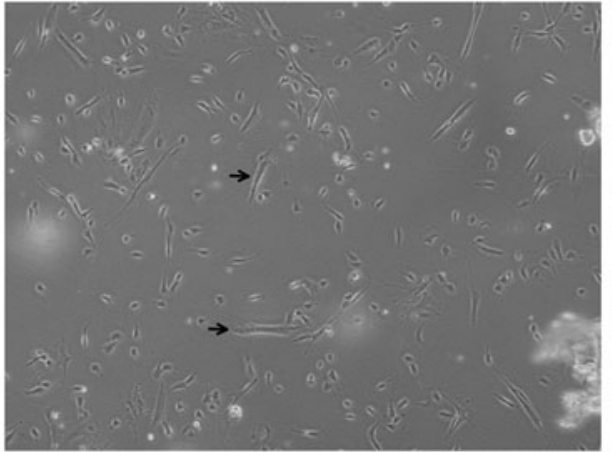

C

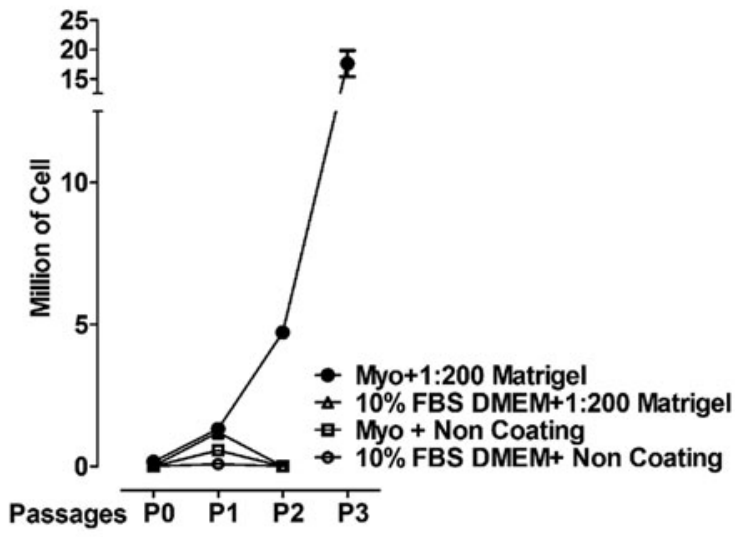

E

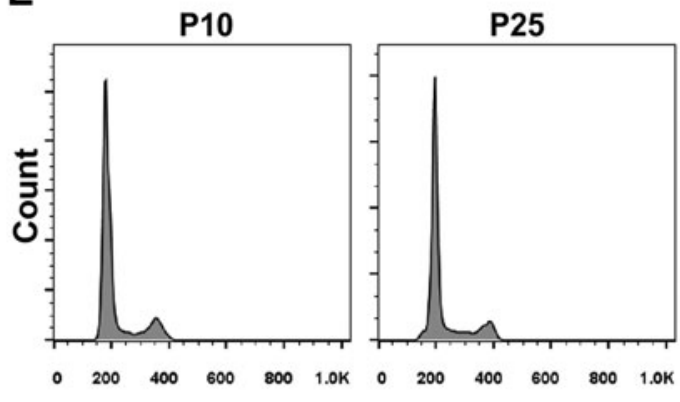

P22

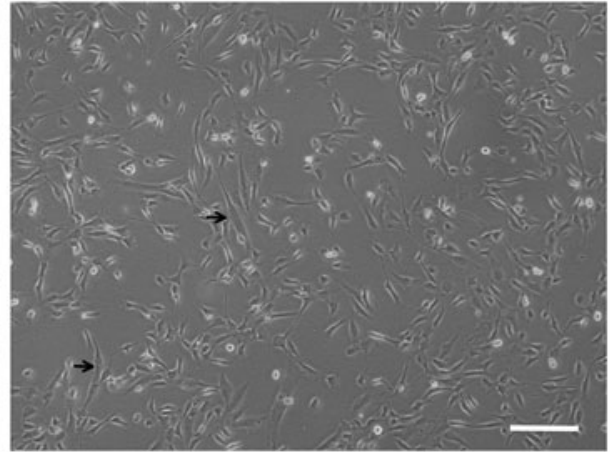

FIG. 1. Comparison of cell culture condition for murine MPC (mMPC) expansion in vitro. (A) Myotube formation by MPCs cultured under five different conditions. (B) Quantification of myotube formation. The data are presented as the number of pixels corresponding to total myotube length per image, as described in Methods ( $n=4$ cultures/condition). (C) Number of MPCs cultured under four different culture conditions, in passages $0-3(n=4$ individual cultures/condition). (D) Number of MPCs, cultured form whole limb muscle tissues of one mouse under condition II, in passages $0-10$ ( $n=4$ cultures). (E) Cell cycle analysis using flow cytometry for expanded MPCs at passages 10 (P10) and 25 (P25). (F) Morphology of in vitro expanded MPCs at low (P0) and high passage (P22). Arrows indicate spontaneous myotube formation. Scale bar $=$ $200 \mu \mathrm{m}$. Data are expressed as mean \pm SD. ${ }^{*} p<0.05$. ns, not significant; MPC, muscle precursor/progenitor cell; DMEM, Dulbecco's modified Eagle's medium; FBS, fetal bovine serum. 
A
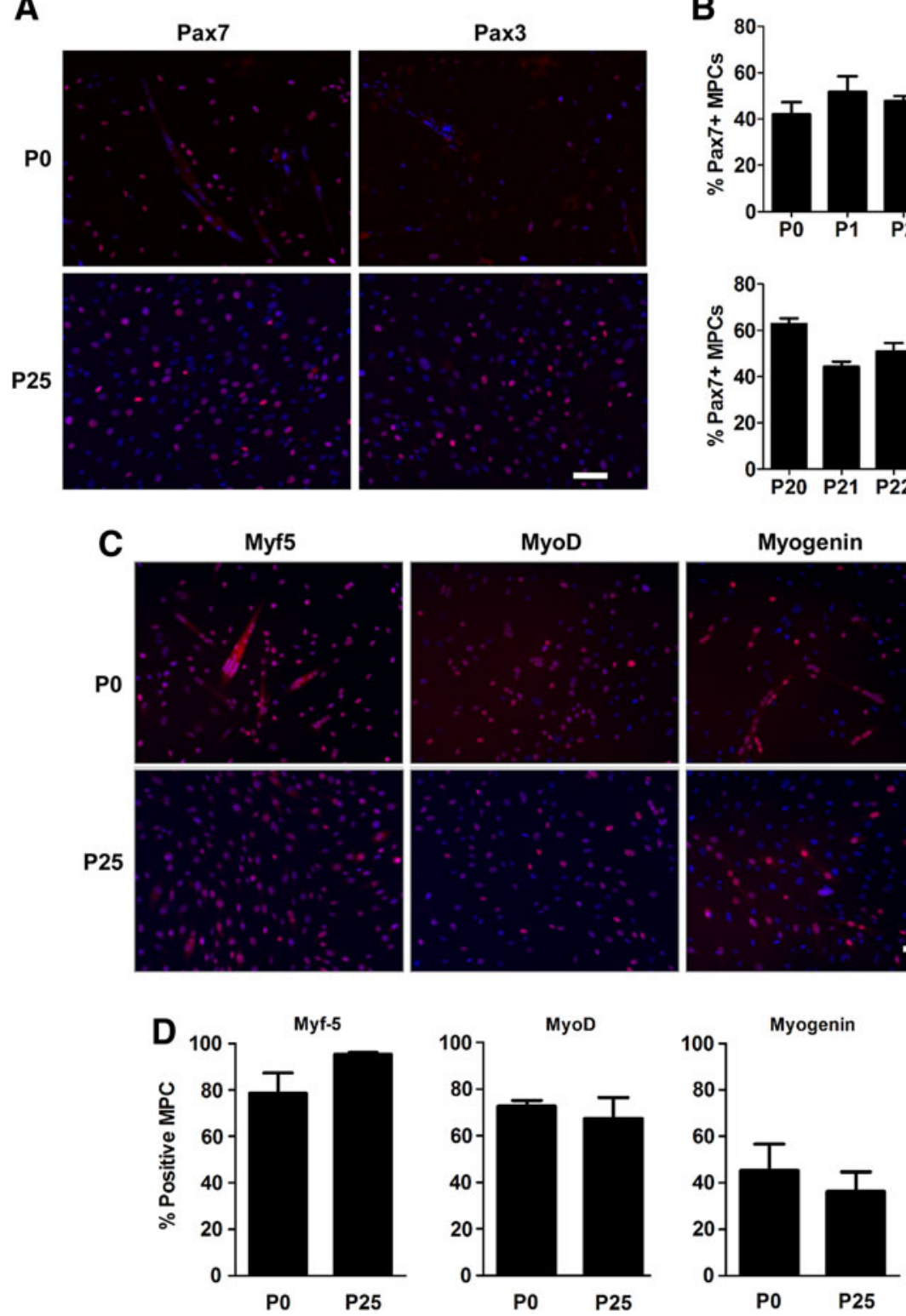

B
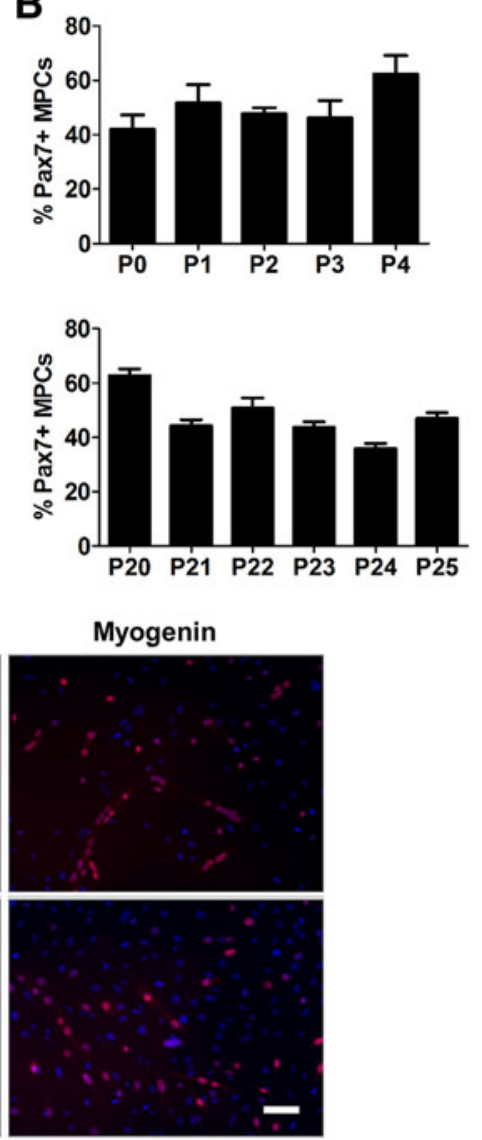

P0 P25
FIG. 2. Long-term in vitro expanded mMPC express muscle stem cell markers. (A) Pax7 and Pax 3 expression in expanded MPCs at passage $0(\mathrm{P} 0)$ and $25(\mathrm{P} 25)$. Cells were stained with antibodies against $\operatorname{Pax} 7$ and $\mathrm{Pax} 3$ (red) and cell nuclei were stained with DAPI (blue). Scale bar $=100 \mu \mathrm{m}$. (B)

Quantification of Pax7 expression (as shown in A) in passages 0-4 (P0-P4) and 20-25 (P20-P25) of MPCs. Results are presented as the frequency of Pax7-positive cells (mean $\pm \mathrm{SD}$ ). (C)

Myf-5, MyoD, and Myogenin expression in expanded MPCs at P0 and P25. Cells were stained with antibody against Myf5, MyoD, and Myogenin (red) and cell nuclei were stained with DAPI (blue). Scale bar $=100$ $\mu \mathrm{m}$. (D) Quantification of Myf-5, MyoD, and Myogenin expression in $\mathrm{P} 0$ and $\mathrm{P} 25$ of MPCs (as shown in C).

Results are presented as the frequency of Myf-5-, MyoD-, and Myogenin-positive cells (mean \pm SD). DAPI, 4',6diamidino-2-phenylindole.

\section{Expanded mMPC engrafted in an injured muscle}

To test the capacity of in vitro expanded mMPCs to engraft in an injured muscle, we performed cell transplantation experiments, using GFP-expressing mMPCs in a cardiotoxin muscle injury mouse model. Murine $\mathrm{GFP}^{+}$MPCs at passages 2,5 , and 10 were injected $24 \mathrm{~h}$ after cardiotoxin injection, and the TA muscles were harvested for analyses 28 days later. Large clusters of donor-derived $\mathrm{GFP}^{+}$myofibers, with centrally localized nuclei were observed (Fig. 4A, B). The $\mathrm{GFP}^{+}$myofibers exhibited heterogeneity in fluorescence intensity (Fig. 4A, arrows), suggesting mosaic regenerating myofibers (Fig. 4Bc), possibly due to heterogeneous fusion of donor cells with host myofibers, consistent with previous findings. ${ }^{16}$

To quantify the engraftment efficiency of in vitro expanded mMPCs, we calculated the RI of transplanted cells. RI was defined as the number of $\mathrm{GFP}^{+}$myofibers per $1 \times 10^{5}$ transplanted mMPCs. ${ }^{25}$ We found a similar RI of up to 80 for mMPCs at passages 2, 5, and 10 (Fig. 4C), suggesting that under our culture conditions mMPCs did not lose their engraftment capacity for at least 10 passages in vitro.

\section{Discussion}

In the current study we described a culture method that enables mMPCs to exhibit robust regenerative capacity during long-term culture, evidenced by their expression of muscle progenitor markers and their ability to self-renew and regenerate myotubes in vitro and myofibers in vivo. The mMPCs could be expanded to trillions of cells and their myogenic capabilities were validated in multiple passages in vitro (up to 25 passages) and in vivo (up to 10 passages), implying that they may have an immediate potential for clinical use.

Conventional protocols for myogenic cell culture often require very young-aged donors ${ }^{4,16,29}$ or allow for very limited cell expansion due to the short period of culture time. ${ }^{25,30-33}$ Using the culture conditions described here, from about $3 \mathrm{~g}$ 
A

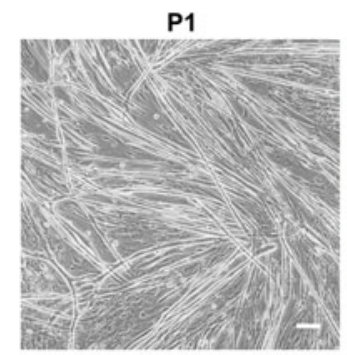

FIG. 3. Myotube formation in expanded mMPCs. (A) In vitro myotube formation in passages 1 (P1), 10 (P10), and 25 (P25) of MPCs. Images were taken at day 7 after cell seeding. Scale bar $=100$ $\mu \mathrm{m}$. (B) Desmin expression in the myotubes was assed using antidesmin antibodies (red) and cell nuclei were stained with DAPI (blue). Right panel shows a representative image of a single myotube showing striations. Scale bar $=100$ $\mu \mathrm{m}$. (C) Quantification of myotubes at multiple passages (P1-P11 and $\mathrm{P} 25)$. Results are presented as the percentage of myotubes with $\geq 5$ nuclei/myotube (mean $\pm \mathrm{SD}$ ).

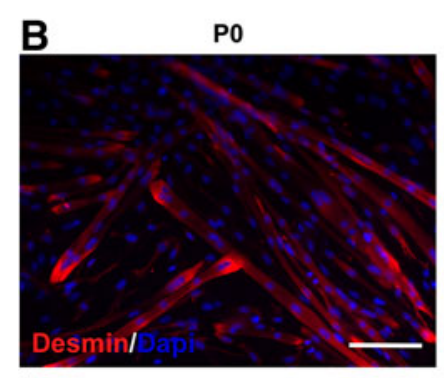

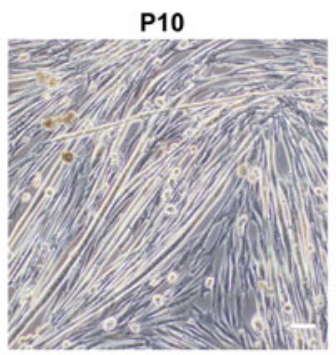
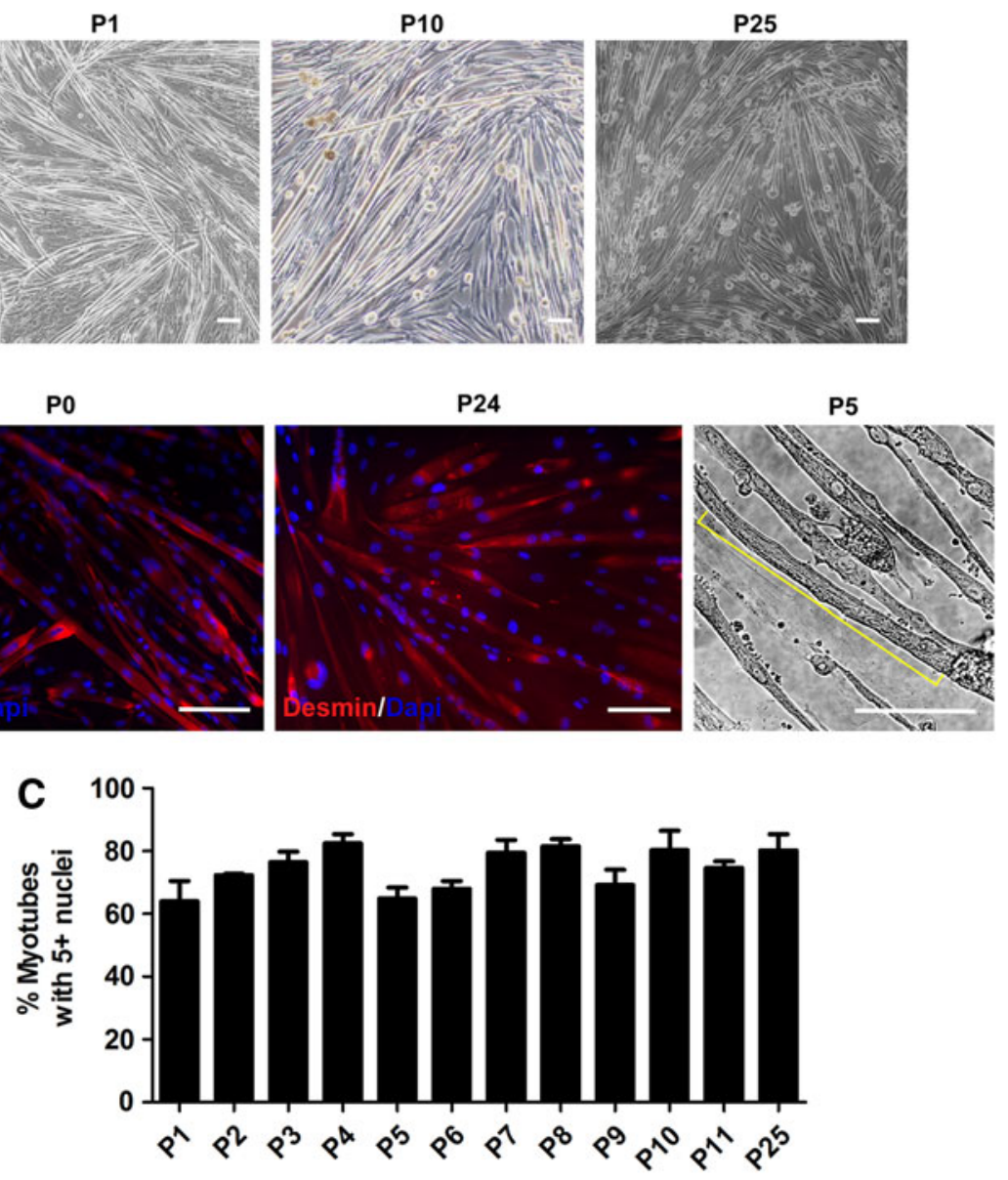

of limb muscle tissue of one adult mouse we were able to obtain one trillion $\left(10^{12}\right)$ myogenic progenitors with engraftment capability. A combination of culture medium containing serum (20\% bovine and $10 \%$ horse) and CEE (Myo medium) and coating the culture dish with Matrigel was responsible for functional cell expansion of adult MPCs. It is likely that such medium combination provided proper niche-related regulatory factors for MPC proliferation including soluble growth factors in the $\mathrm{CEE}^{34,35}$ and ECM adhesive proteins in the Matrigel. ${ }^{36-39}$ Despite of the acknowledgment of the beneficial effects of CEE and Matrigel separately in MPC culture, there are no reports that documented the combination effect of these two reagents on long-term self-renewal of adult MPCs. Future studies are needed to identify the specific adhesive proteins and growth factors in the culture system reported here, which were responsible for the robust MPC expansion and maintenance of myogenic capacities.

Expression of Pax 7 and Pax3 in MPCs is a good predictor for their engraftment in vivo, ${ }^{4,16,17,26}$ whereas the loss of expression of either of them results in decreased muscle regeneration. $^{26,27,40}$ In vitro culture leads to rapid and significant decline in the expression of myogenic progenitor markers such as Pax3/7 and engraftment capacities. ${ }^{14,17}$ To the contrary, in vitro expanded mMPCs in this study showed consistent engraftment capacity, indicated by an RI up to 80. This implies the expanded MPCs at passage 10 can potentially re- generate 800 million $\left(8 \times 10^{8}\right)$ myofibers, sufficient to regenerate the skeletal muscles in the whole human body. Furthermore, there is no statistical difference in engraftment between cells injected in Matrigel (1:5) or in PBS $+2 \%$ FBS (data not shown). We were not able to test the engraftability of mMPCs cultured under the control conditions (III, IV, and V, Fig. 1B). Not only because the cells lost Pax7 expression, they also failed to grow beyond passage 3 (Fig. 3C) and we did not have enough cells for these experiments.

Common muscle regeneration models (e.g., dystrophic or post-injury radiation-treated mice) have significantly low numbers of endogenous muscle stem cells. The nude mouse model used in the current study has a normal size of endogenous muscle stem cell pool, which implies that implanted MPCs had to compete for engraftment with endogenous muscle stem cells. Thus, in vitro expanded MPCs will theoretically have a greater impact on regeneration in muscles of dystrophic recipients. Although embryonic stem cells ${ }^{41}$ and induced pluripotent stem cells are promising cell sources for muscle therapy, ${ }^{42}$ the potential of tumorigenesis limits their clinical use. ${ }^{43}$ In vitro expanded mMPCs did not show any signs of tumorigenesis after implantation (data not shown), which is different from the tumorigenesis potential in a former study using C2C12 cell line for cell therapy, ${ }^{44}$ suggesting they could be a safe option for clinical application.

In summary, we described a tissue culture system for generating a large number of mMPCs with adequate myogenic 
A P2 (50X)
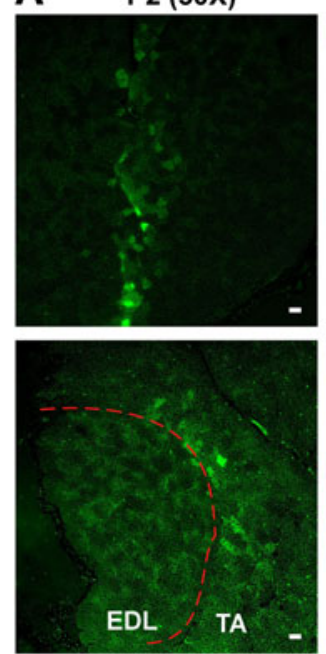

P5 (200X)
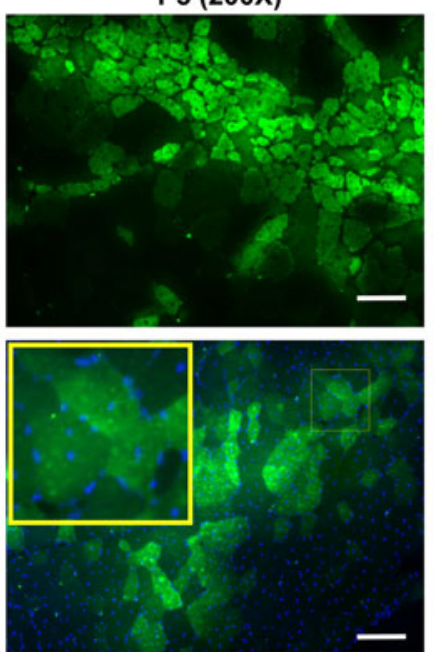
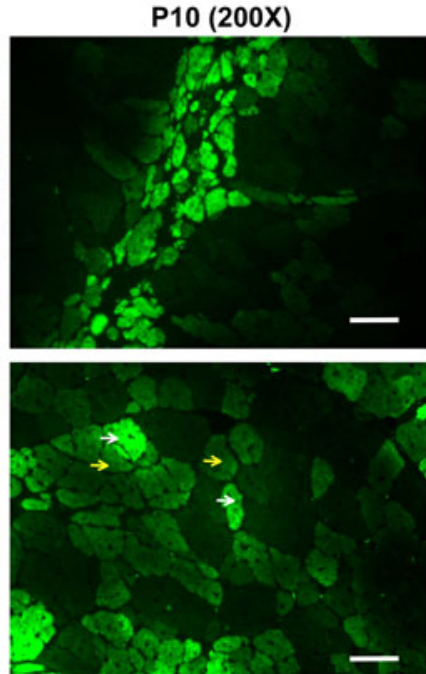

B
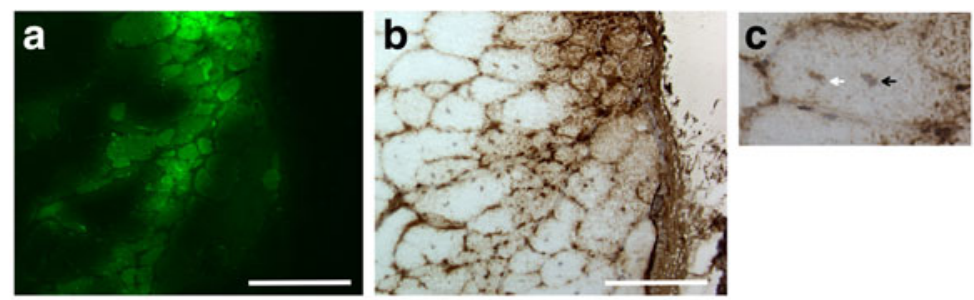

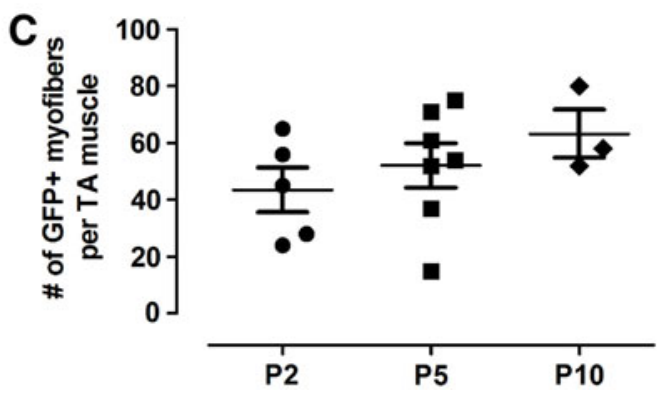

FIG. 4. Engraftment of in vitro expanded $\mathrm{mMPC}$ engraft into injured muscle tissue. (A) Nude mice transplanted with $1 \times 10^{5} \mathrm{GFP}^{+}$ mMPCs at passage 2,5 , and $10(\mathrm{P} 2$, $\mathrm{P} 5$, and $\mathrm{P} 10$, respectively), as described in Methods. Tibialis anterior (TA) muscles were harvested 4 weeks after transplantation and processed for fluorescence imaging (epifluorescence). Cross-section of the TA muscles showed large clusters of $\mathrm{GFP}^{+}$myofibers with centrally localized nuclei (yellow box). Dashed red line (left lower panel) indicates the border between the epifluorescent signal of $\mathrm{GFP}^{+}$ myofibers and the autofluorescence of the adjacent host extensor digitorumlongus (EDL) muscles. White and yellow arrows indicated heterogeneous epifluorescence of $\mathrm{GFP}^{+}$myofibers. Scale bar $=100$ $\mu \mathrm{m}$. (B) Detection of GFP in engrafted muscle. GFP was detected in serial sections of the muscle tissue by epifluorescence (green, a) and staining with anti-GFP antibodies (brown staining, b), showing similar patterns of GFP signal. c. A chimeric myofiber contains host (blue nucleus, black arrow) and donor (brown nucleus, white arrow) nuclei, as determined by staining with anti-GFP antibodies. (C) Quantification of $\mathrm{GFP}^{+}$myofibers. The number of $\mathrm{GFP}^{+}$myofibers was determined in 100-200 serial sections of each TA muscle. GFP, green fluorescent protein.

capabilities after long-term culture in vitro. Our culture system can potentially be adapted for the expansion of hMPCs to generate high enough numbers for the treatment of a broad range of muscle degenerative diseases in human patients.

\section{Acknowledgments}

We thank Dr. Giuseppe Orlando from Wake Forest Institute for Regenerative Medicine for his assistance in accessing human muscle tissue. The Pax7, Pax3, and Myogenin antibodies (developed by Astushi Kawakami and Woodring E. Wright, respectively) were obtained from the Developmental Studies Hybridoma Bank developed under the auspices of the National Institute of Child Health and Human Development and maintained by the University of Iowa, Department of Biology (Iowa City, IA). This work is supported by AFIRM Compartment Syndrome project (W81XWH-08-2-0032-SCSTH).

\section{Author Disclosure Statement}

The authors declare they have no potential conflicts of interest.

\section{References}

1. Vandenburgh H, Shansky J, Benesch-Lee F, et al. Automated drug screening with contractile muscle tissue engineered from dystrophic myoblasts. FASEB J. 2009;23: 3325-3334.

2. Grosberg A, Nesmith AP, Goss JA, et al. Muscle on a chip: in vitro contractility assays for smooth and striated muscle. J Pharmacol Toxicol Methods. 2012;65:126-135.

3. Collins CA, Olsen I, Zammit PS, et al. Stem cell function, self-renewal, and behavioral heterogeneity of cells from the adult muscle satellite cell niche. Cell. 2005;122:289-301.

4. Montarras D, Morgan J, Collins C, et al. Direct isolation of satellite cells for skeletal muscle regeneration. Science. 2005;309:2064-2067.

5. Kinoshita I, Vilquin JT, Tremblay JP. Mechanism of increasing dystrophin-positive myofibers by myoblast transplantation: study using $\mathrm{mdx} / \mathrm{beta}-$ galactosidase transgenic mice. Acta Neuropathol. 1996;91:489-493.

6. Skuk D, Goulet M, Roy B, et al. Dystrophin expression in muscles of duchenne muscular dystrophy patients after high-density injections of normal myogenic cells. J Neuropathol Exp Neurol. 2006;65:371-386. 
7. Tedesco FS, Dellavalle A, Diaz-Manera J, et al. Repairing skeletal muscle: regenerative potential of skeletal muscle stem cells. J Clin Invest. 2010;120:11-19.

8. Tremblay JP, Skuk D. Another new "super muscle stem cell" leaves unaddressed the real problems of cell therapy for duchenne muscular dystrophy. Mol Ther. 2008;16:1907-1909.

9. Nawroth JC, Lee H, Feinberg AW, et al. A tissue-engineered jellyfish with biomimetic propulsion. Nat Biotechnol. 2012; 30:792-797.

10. Scadden DT. The stem-cell niche as an entity of action. Nature. 2006;441:1075-1079.

11. Cosgrove BD, Sacco A, Gilbert PM, et al. A home away from home: challenges and opportunities in engineering in vitro muscle satellite cell niches. Differentiation. 2009; 78:185-194.

12. Kuang S, Gillespie MA, Rudnicki MA. Niche regulation of muscle satellite cell self-renewal and differentiation. Cell Stem Cell. 2008;2:22-31.

13. Wagers AJ. The stem cell niche in regenerative medicine. Cell Stem Cell. 2012;10:362-369.

14. Gilbert PM, Havenstrite KL, Magnusson KE, et al. Substrate elasticity regulates skeletal muscle stem cell self-renewal in culture. Science. 2010;329:1078-1081.

15. Parker MH, Loretz C, Tyler AE, et al. Activation of Notch signaling during ex vivo expansion maintains donor muscle cell engraftment. Stem Cells. 2012;30:2212-2220.

16. Cerletti M, Jurga S, Witczak CA, et al. Highly efficient, functional engraftment of skeletal muscle stem cells in dystrophic muscles. Cell. 2008;134:37-47.

17. Sacco A, Doyonnas R, Kraft P, et al. Self-renewal and expansion of single transplanted muscle stem cells. Nature. 2008;456:502-506.

18. Rodin S, Domogatskaya A, Strom S, et al. Long-term selfrenewal of human pluripotent stem cells on human recombinant laminin-511. Nat Biotechnol. 2010;28:611-615.

19. Melkoumian Z, Weber JL, Weber DM, et al. Synthetic peptide-acrylate surfaces for long-term self-renewal and cardiomyocyte differentiation of human embryonic stem cells. Nat Biotechnol. 2010;28:606-610.

20. Villa-Diaz LG, Nandivada H, Ding J, et al. Synthetic polymer coatings for long-term growth of human embryonic stem cells. Nat Biotechnol. 2010;28:581-583.

21. Eberli D, Soker S, Atala A, et al. Optimization of human skeletal muscle precursor cell culture and myofiber formation in vitro. Methods. 2009;47:98-103.

22. Okabe M, Ikawa M, Kominami K, et al. 'Green mice' as a source of ubiquitous green cells. FEBS Lett. 1997;407: 313-319.

23. Jackson KA, Snyder DS, Goodell MA. Skeletal muscle fiber-specific green autofluorescence: potential for stem cell engraftment artifacts. Stem Cells. 2004;22:180-187.

24. Swenson ES, Price JG, Brazelton T, et al. Limitations of green fluorescent protein as a cell lineage marker. Stem Cells. 2007;25:2593-2600.

25. Deasy BM, Lu A, Tebbets JC, et al. A role for cell sex in stem cell-mediated skeletal muscle regeneration: female cells have higher muscle regeneration efficiency. J Cell Biol. 2007; 177:73-86.

26. Relaix F, Rocancourt D, Mansouri A, et al. A Pax3/Pax7dependent population of skeletal muscle progenitor cells. Nature. 2005;435:948-953.

27. Seale P, Sabourin LA, Girgis-Gabardo A, et al. Pax7 is required for the specification of myogenic satellite cells. Cell. 2000;102:777-786.
28. Sambasivan R, Yao R, Kissenpfennig A, et al. Pax7expressing satellite cells are indispensable for adult skeletal muscle regeneration. Development. 2011;138:3647-3656.

29. Ehrhardt J, Brimah K, Adkin C, et al. Human muscle precursor cells give rise to functional satellite cells in vivo. Neuromuscul Disord. 2007;17:631-638.

30. Rando TA, Blau HM. Primary mouse myoblast purification, characterization, and transplantation for cell-mediated gene therapy. J Cell Biol. 1994;125:1275-1287.

31. Qu-Petersen Z, Deasy B, Jankowski R, et al. Identification of a novel population of muscle stem cells in mice: potential for muscle regeneration. J Cell Biol. 2002;157:851-864.

32. Keire P, Shearer A, Shefer G, et al. Isolation and culture of skeletal muscle myofibers as a means to analyze satellite cells. Methods Mol Biol. 2013;946:431-468.

33. Shefer G, Van de Mark DP, Richardson JB, et al. Satellitecell pool size does matter: defining the myogenic potency of aging skeletal muscle. Dev Biol. 2006;294:50-66.

34. Ii I, Kimura I, Ozawa E. A myotrophic protein from chick embryo extract: its purification, identity to transferrin, and indispensability for avian myogenesis. Dev Biol. 1982;94: 366-377.

35. Toyota N, Shimada Y. Isoform variants of troponin in skeletal and cardiac muscle cells cultured with and without nerves. Cell. 1983;33:297-304.

36. Kleinman HK, Martin GR. Matrigel: basement membrane matrix with biological activity. Semin Cancer Biol. 2005; 15:378-386.

37. Domogatskaya A, Rodin S, Boutaud A, et al. Laminin-511 but not $-332,-111$, or -411 enables mouse embryonic stem cell self-renewal in vitro. Stem Cells. 2008;26:28002809.

38. Yao CC, Ziober BL, Sutherland AE, et al. Laminins promote the locomotion of skeletal myoblasts via the alpha 7 integrin receptor. J Cell Sci. 1996;109(Pt 13):3139-3150.

39. Denzer AJ, Schulthess T, Fauser C, et al. Electron microscopic structure of agrin and mapping of its binding site in laminin-1. EMBO J. 1998; 17:335-343.

40. Lepper C, Partridge TA, Fan CM. An absolute requirement for Pax7-positive satellite cells in acute injury-induced skeletal muscle regeneration. Development. 2011;138:36393646.

41. Darabi R, Gehlbach K, Bachoo RM, et al. Functional skeletal muscle regeneration from differentiating embryonic stem cells. Nat Med. 2008;14:134-143.

42. Darabi R, Arpke RW, Irion S, et al. Human ES- and iPSderived myogenic progenitors restore DYSTROPHIN and improve contractility upon transplantation in dystrophic mice. Cell Stem Cell. 2012;10:610-619.

43. Ben-David U, Benvenisty N. The tumorigenicity of human embryonic and induced pluripotent stem cells. Nat Rev Cancer. 2011;11:268-277.

44. Wernig A, Irintchev A, Hartling A, et al. Formation of new muscle fibres and tumours after injection of cultured myogenic cells. J Neurocytol. 1991;20:982-997.

Address correspondence to: Shay Soker, PhD

Wake Forest Institute for Regenerative Medicine Wake Forest School of Medicine Medical Center Boulevard Winston Salem, NC 27157

E-mail: ssoker@wakehealth.edu 


$\begin{aligned} & \text { Abbreviations Used } \\ \mathrm{CEE} & =\text { chicken embryo extract } \\ \mathrm{DMEM} & =\text { Dulbecco's modified Eagle's medium } \\ \mathrm{ECM} & =\text { extracellular matrix } \\ \mathrm{FBS} & =\text { fetal bovine serum } \\ \mathrm{GFP} & =\text { green fluorescent protein } \\ \mathrm{hMPC} & =\text { human muscle precursor/progenitor cell }\end{aligned}$

$\begin{aligned} \mathrm{mMPC} & =\text { murine muscle precursor/progenitor cell } \\ \mathrm{MPC} & =\text { muscle precursor/progenitor cell } \\ \text { Myo medium } & =\text { myogenic medium } \\ \mathrm{NIH} & =\text { National Institutes of Health } \\ \mathrm{PBS} & =\text { phosphate-buffered saline } \\ \mathrm{RI} & =\text { regenerative index } \\ \mathrm{TA} & =\text { tibialis anterior }\end{aligned}$

